Article

\title{
Sevoflurane-Sulfobutylether- $\beta$-Cyclodextrin Complex: Preparation, Characterization, Cellular Toxicity, Molecular Modeling and Blood-Brain Barrier Transport Studies
}

\section{Sergey Shityakov ${ }^{1, *}$, István Puskás ${ }^{2}$, Katalin Pápai ${ }^{3}$, Ellaine Salvador ${ }^{1}$, Norbert Roewer ${ }^{1,3}$, Carola Förster ${ }^{1}$ and Jens-Albert Broscheit ${ }^{1,3}$}

1 Department of Anaesthesia and Critical Care, University of Würzburg, 97080 Würzburg, Germany; E-Mails: Salvador_E@ukw.de (E.S.);AN_Direktion@ukw.de (N.R.); Foerster_C@ukw.de (C.F.); Broscheit_J@ukw.de (J.-A.B.)

2 CycloLab Cyclodextrin Research \& Development Laboratory Ltd., H-1097 Budapest, Hungary; E-Mail: puskas@cyclolab.hu

3 Sapiotec Ltd., 97078 Würzburg, Germany; E-Mail: papai@sapiotec.de

* Author to whom correspondence should be addressed; E-Mail: E_Shityako_S@ukw.de; Tel.: +49-931-2013-0016; Fax: +49-931-2013-0019.

Academic Editor: Derek J. McPhee

Received: 12 April 2015 / Accepted: 25 May 2015 / Published: 3 June 2015

\begin{abstract}
The objective of the present investigation was to study the ability of sulfobutylether- $\beta$-cyclodextrin (SBE $\beta C D$ ) to form an inclusion complex with sevoflurane (SEV), a volatile anesthetic with poor water solubility. The inclusion complex was prepared, characterized and its cellular toxicity and blood-brain barrier (BBB) permeation potential of the formulated SEV have also been examined for the purpose of controlled drug delivery. The SEV-SBE $\beta C D$ complex was nontoxic to the primary brain microvascular endothelial (pEND) cells at a clinically relevant concentration of sevoflurane. The inclusion complex exhibited significantly higher BBB permeation profiles as compared with the reference substance (propranolol) concerning calculated apparent permeability values $\left(\mathrm{P}_{\mathrm{app}}\right)$. In addition, SEV binding affinity to $\mathrm{SBE} \beta C D$ was confirmed by a minimal Gibbs free energy of binding ( $\Delta \mathrm{G}_{\text {bind }}$ ) value of $-1.727 \pm 0.042 \mathrm{kcal} \cdot \mathrm{mol}^{-1}$ and an average binding constant $\left(\mathrm{K}_{\mathrm{b}}\right)$ of $53.66 \pm 9.24 \mathrm{mM}$ indicating rapid drug liberation from the cyclodextrin amphiphilic cavity.
\end{abstract}


Keywords: cyclodextrin formulations; sevoflurane; sulfobutylether- $\beta$-cyclodextrin; blood-brain barrier; primary microvascular endothelial cells; molecular docking; molecular liphophilicity potential

\section{Introduction}

Sevoflurane (SEV, fluoromethylhexafluoroisopropyl ether), is an inhalational anesthetic recommended for almost 40 years for induction and maintenance of general anesthesia [1]. SEV has become one of the most commonly used inhaled anesthetic agents due to its favorable therapeutic profile. However, because of its volatile properties, high lipophilicity, and poor aqueous solubility (experimental hydrophobicity $(\log \mathrm{P}): 2.4)$, the drug cannot be administered orally or via intravenous injection. Because of the low solubility of SEV in the blood (blood/gas partition coefficient (Sblood/gas): 0.63-0.69) [2], a minimal amount of SEV is required to be dissolved in the blood before the alveolar partial pressure is in equilibrium with the arterial partial pressure. Therefore, there is a rapid increase in the alveolar concentration $\left(\mathrm{F}_{\mathrm{A}}\right)$ of this anesthetic toward the inspired concentration during induction. This will impose some difficulties on the SEV bioavailability as a result of the insufficient $\mathrm{F}_{\mathrm{A}}$ rate, which could be improved via an intravenously injectable formulation of SEV with hydrophilic/amphiphilic cyclodextrins (CDs), including sevoflurane-sulfobutylether- $\beta$-cyclodextrin (SBE $\beta C D$ ). On the other hand, improved bioavailability and the blood-brain barrier (BBB) permeability of SEV may also lead to a dose reduction and thus might abolish the formation of some chemically active metabolites, such as compound A, which is believed to be responsible for kidney damage [3,4]. However, still today, the potential of SBE $\beta C D$ as an excipient in improving the therapeutic efficacy and diminishing side-effects of SEV has not yet been determined.

SBE $\beta C D$ is a solubilizing agent for poorly water-soluble compounds used in the formulation of both solid dosage and parenteral forms [5]. The torus-like structure of the highly water-soluble SBE $\beta C D$ molecules consists of a hydrophobic internal cavity that enables the formation of a reversible drug-CD complex and highly hydrophilic exterior interface [6]. The aqueous solubility of SBEßCD ( 70\%) is significantly higher than that of the parental $\beta$-CD form, which only has $1.85 \%$ at $25{ }^{\circ} \mathrm{C}$ [7]. Furthermore, SBE $\beta C D$ does not exhibit the nephrotoxicity associated with $\beta$-cyclodextrin [8]. Moreover, no cytotoxic effects of SBE $\beta C D$ on heterogeneous human epithelial colorectal adenocarcinoma (Caco-2) cells [9] have been detected due to its minimal capacity to solubilize cholesterol and other membrane lipids $[10,11]$. It has been previously demonstrated that SBE $\beta C D$ possesses no cytotoxic effect on heterogeneous human epithelial colorectal adenocarcinoma (Caco-2) cells [9] and has a minimal capacity to solubilize cholesterol and other membrane lipids [10]. Additionally, in vitro and in vivo the anti-hemolytic potencies of SBE $\beta C D$ have been detected in the previous studies during its direct exposure to cells or in intravenous applications $[8,12]$. In view of this, SBE $\beta C D$ can be considered a safe formulating agent for oral and intravenous administration. SBE $\beta C D$ inclusion complexes of different anesthetics such as alphaxalone, propofol, and etomidate have already been evaluated, showing their potential to improve the pharmacokinetics and pharmacodynamics of drug molecules [12-15]. 
In the present investigation, the formation of an inclusion complex between SEV and SBE $\beta C D$ using the industrially feasible optimized formulation procedure was studied. The SEV-SBE $\beta C D$ complex was characterized by various methods to assess its chemical stability, cell toxicity, and BBB permeation. Furthermore, we also analyzed the interaction of SEV and SBE $\beta C D$ using the molecular docking technique to predict the SEV binding affinity to cyclodextrin and to define the factors responsible for the drug release kinetics.

\section{Results and Discussion}

In the first phase of the investigations, the SBE $\beta C D$ and SEV-SBE $\beta C D$ compounds were investigated by X-ray powder diffractometry and polarized light microscopy (only for the complex) to verify the amorphous or crystalline character of the studied compounds. Before this, the coarse-grained, porous macroscopic structure of the solid-state lyophilized SEV-SBE $\beta C D$ complex was observed as semi-uniform with concentric porosity (Figure S1, Supplementary Material). The staggered and sponge-like pattern of its surface topography without any periodicity in the mm-scale, and the concentric distribution of the pores with large height values are displayed in Figure 1A-D.

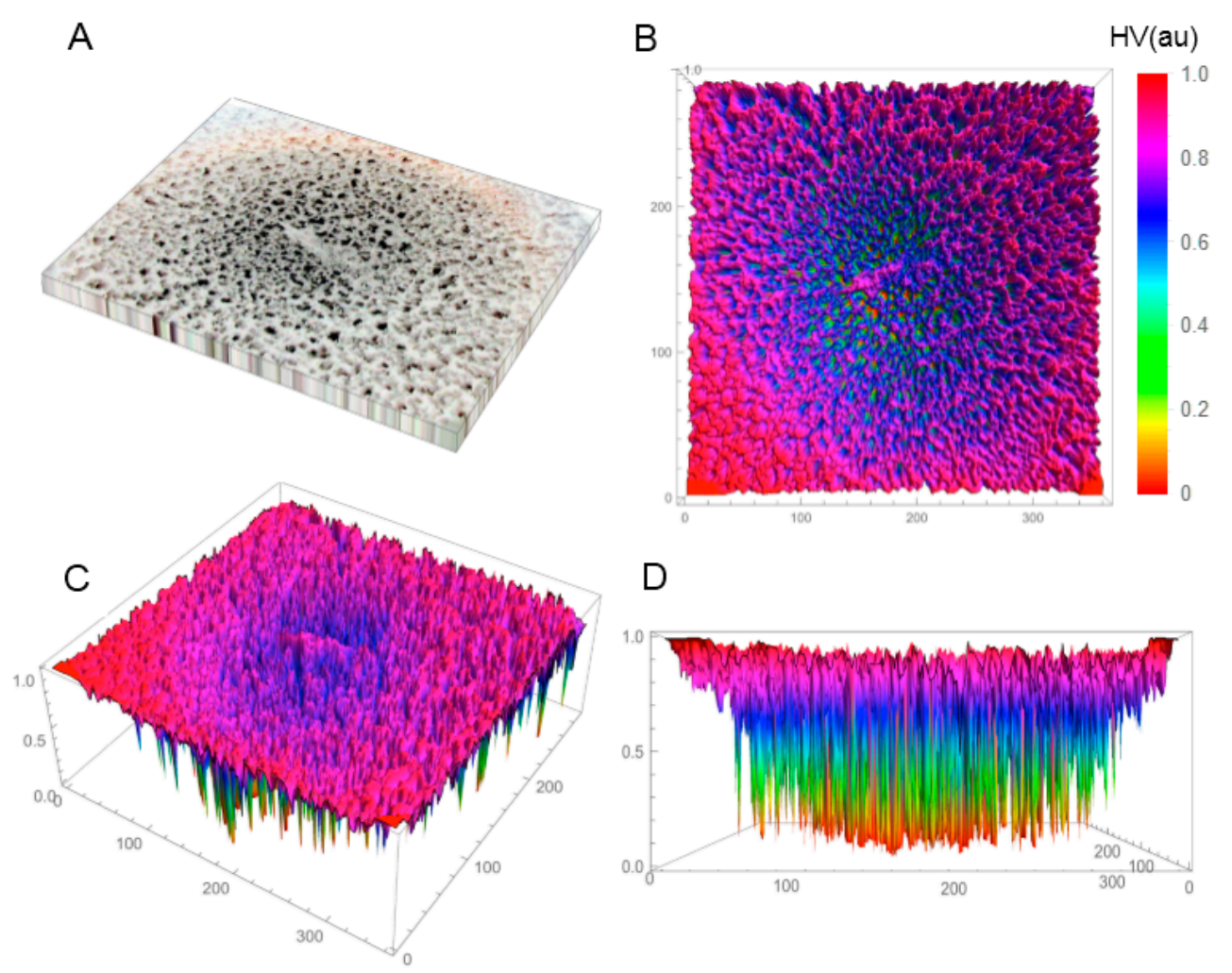

Figure 1. Reconstructed 3D structure of the solid-state lyophilized SEV-SBE $\beta C D$ before grinding taken by a normal digital camera (A); The top (B); default (C); and front (D) view of the SEV-SBE $\beta C D$ surface 3D plot was generated to evaluate and visualize the porosity using the array of height values (HV) measured in arbitrary units (au) with hue color function. The substance was in a round bottom flask, and the material in the photo represents an approximately $50 \times 40 \mathrm{~mm}$ area. 
To produce a powder, the solid bulk complex was ground and sieved through a $0.3 \mathrm{~mm}$ mesh sieve. The lyophlization process was maintained for $24 \mathrm{~h}$ with a chamber temperature of $-50{ }^{\circ} \mathrm{C}$ and pressure of 45-65 mTorr (6.0-8.7 Pa).

Powder X-ray diffractometry is a useful method for the detection of cyclodextrin complexation in powder or microcrystalline states [16]. The diffraction pattern of the drug-CD complex should be clearly distinct from that of the superimposition of each of the components if a true inclusion complex is formed [17]. By comparing the X-ray diffraction patterns, the different phases present at room temperature could be identified for each compound. In the X-ray diffractograms of the analyzed compounds, it is possible to observe broad peaks of different beam intensities ( 225 and 325 cps) at the diffraction angles $(2 \theta)$ of $19.0^{\circ}$ and $19.5^{\circ}$, indicating the amorphous structure of SBE $\beta C D$ (cyclodextrin with electron-donating side chains) and its complex (Figure 2A). Moreover, the amorphous and homogeneous SEV-SBE $\beta C D$ appearance was also confirmed under polarized light, as no light interference patterns were observed (Figure 2B). At this stage of the study, the powder diffraction patterns offered no stoichiometry information on SEV inclusion in the internal cyclodextrin cavity. However, it is likely that the presence of substituents extends the CD cavity length, and therefore possibly favors the occurrence of hydrogen bonds with the guest molecule [18]. The formation of the amorphous state may be attributed mainly to the random number of sulfobutyl ether groups per cyclodextrin molecule [7], which was observed in some experimental studies to investigate its effect on accelerated degradation of the complexed drug [19].
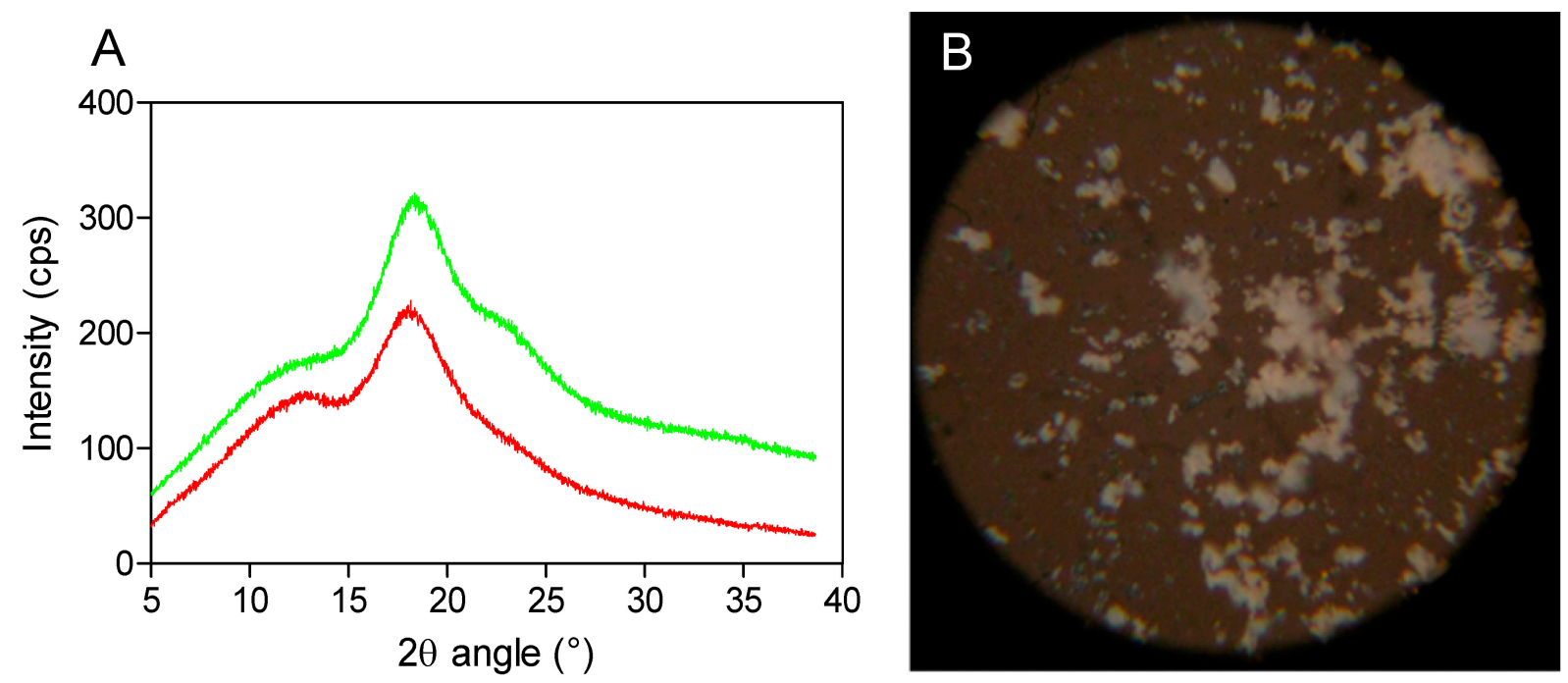

Figure 2. X-ray powder diffraction patterns (A) as red $(\mathrm{SBE} \beta C D)$ and green curves (SEV-SBE $\beta C D)$ and polarized light microscopy (B) of the SEV-SBE $\beta C D$ complex at 100× magnification. The beam intensity is measured in counts per second, abbreviated as cps.

In the next step, the practically identical electropherograms obtained for the two analyzed substances showed that the multicomposite structure of the SEV-SBEßCD molecule remained intact throughout the complex preparation method (Figure 3A,B). In addition, the accelerated stability testing after two weeks at $40{ }^{\circ} \mathrm{C}$ in an open container using gas chromatography also showed the strong supramolecular interaction between the SEV and SBE $\beta C D$ components of the complex, and that the $\mathrm{w} \%$ value remained the same (8.6\%) over time (unpublished data). Reconstitution of $100 \mathrm{mg}$ of the complex in $0.9 \mathrm{~mL}$ of 
distilled water resulted in a clear solution; the residual moisture content determined according to the volumetric Karl-Fischer titration method [20] was elevated less than $1.2 \%$, from $3.5 \%$ to $4.7 \%$ w/w with a 2 -week storage time. Therefore, a second lyophilization step (warm up in vacuum) was not required due to the resulting relatively low residual water content of the substance.
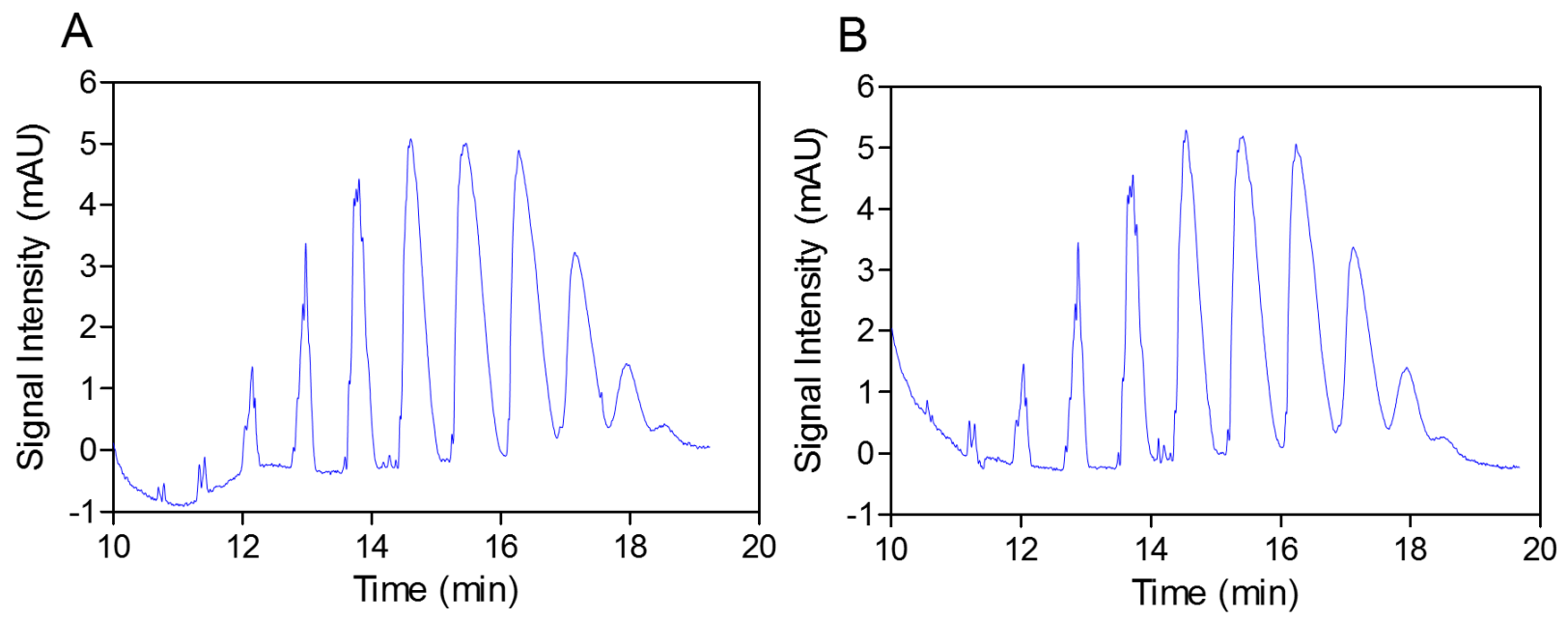

Figure 3. Capillary elecropherograms of the SBE $\beta C D$ compound (A) and SEV-SBE $\beta C D$ complex (B) on a $50 \mathrm{~cm}, 50 \mu \mathrm{m}$ uncoated fused-silica capillary. The signal intensity is measured in milli absorbance units, abbreviated as mAU.

The CellTiter-Glo ${ }^{\circledR}$ luminescent cell viability assay (Promega, Madison, WI, USA) was used to determine the cytotoxicity of the SEV-SBE $\beta C D$ complex taken into consideration the drug clinically relevant concentration of $40 \mu \mathrm{g} \cdot \mathrm{mL}^{-1}$ [21], which corresponds to $465 \mu \mathrm{g} \cdot \mathrm{mL}^{-1}$ of the complex. Cell viability was assessed by the amount of ATP produced by metabolically active cells. The released ATP converts luciferin substrate to luciferin oxide, and released luminescence signals were recorded. The results of this assay showed the absence of toxic effect for SEV-SBEßCD on pEND cells after $24 \mathrm{~h}$ of incubation. Overall, with increased time, no significant difference from the actual cell viability for SEV-SBE $\beta C D$ was detected, and their luminescence levels remained above the median toxic dose (TD 50 ) threshold. A significant reduction in luminescence activity was observed as a sign of a massive cell death at 10\% dimethyl sulfoxide, which served as a positive control (Figure 4).

Using a Transwell ${ }^{\circledR}$ model with $0.4 \mu \mathrm{m}$ pore size and $33.6 \mathrm{~mm}^{2}$ surface area of the polyethylene terephthalate (PET) filter membrane, the BBB permeation rate of the SEV-SBE $\beta C D$ complex was assessed according to the procedure as described in the Experimental section. Prior to this, the dynamic TER measurements of the pEND monolayers and FITC-labeled dextran $(4 \mathrm{kDa})$ permeation assay were taken to validate the cellular tightness and provide paracellular property information.

The cells exhibited TER values ranging from $165.67 \pm 2.08$ before and $153 \pm 3.46 \Omega \cdot \mathrm{cm}^{2}$ after $24 \mathrm{~h}$ of incubation with SEV-SBE $\beta C D$ in comparison to the control group with the TER values in the range from $94.67 \pm 2.08$ to $92.67 \pm 1.53 \Omega \cdot \mathrm{cm}^{2}$ (Figure $5 \mathrm{~A}$ ). To evaluate the paracellular permeation through the tight junctions, the apical-to-basolateral flux of FITC-dextran was measured across the pEND monolayers. The FITC-dextran flux was significantly higher in the control group (128.33 \pm 7.64 and $113 \pm 26.63 \mathrm{RFU})$ than that of pEND cells $(32.33 \pm 3.79$ and $48.33 \pm 6.43 \mathrm{RFU})$ after 30 min of incubation with FITC-dextran (Figure 5B). 


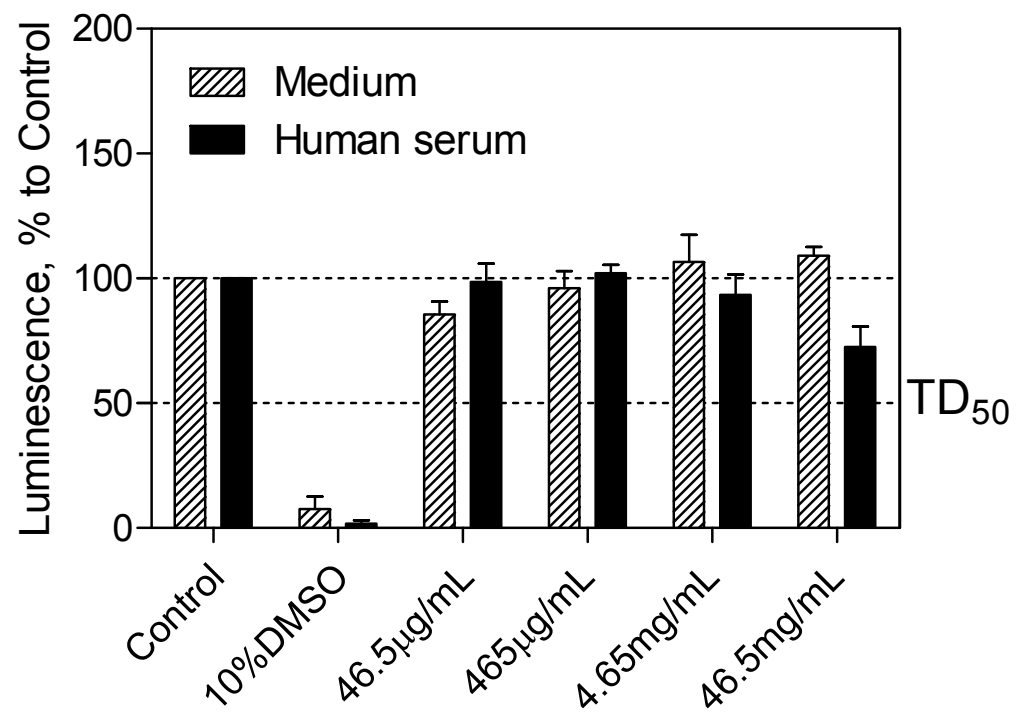

Figure 4. pEND cell viability assay to measure SEV-SBEßCD toxic effect over time $(24 \mathrm{~h})$. The luminescence is measured in percentage to a control group (untreated cells). 10\% solution of DMSO was used as positive control. The median toxic dose level is abbreviated as $\mathrm{TD}_{50}$. The thresholds are depicted as dashed lines. Data represent means \pm standard deviation of three independent experiments
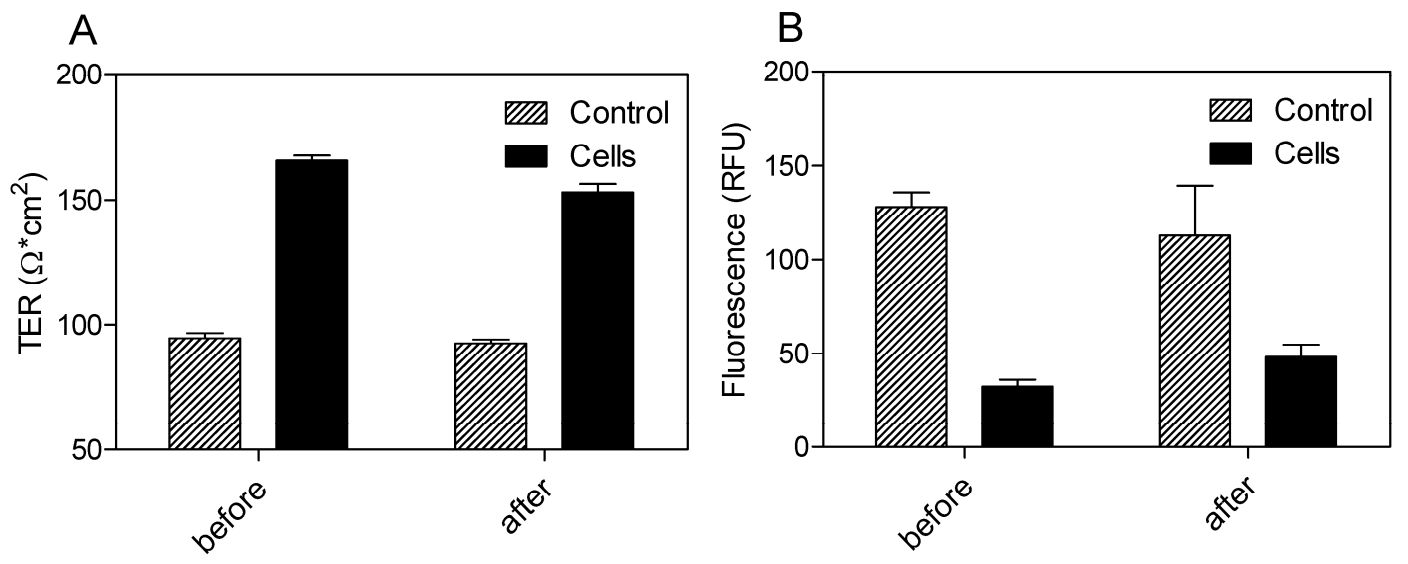

Figure 5. TER measurements (A) and FITC-dextran $(4 \mathrm{kDa})$ permeation assay $(\mathbf{B})$ of propranolol as system validation substance and SEV-SBE $\beta C D$ complex. Collagen 4-coated empty inserts were used as a control. The fluorescence is measured in relative fluorescence units, abbreviated as RFU. Data represent means \pm standard deviation of three independent experiments.

In the next phase of investigations, transport experiments were carried out for all compounds at a concentration of $30 \mu \mathrm{g} \cdot \mathrm{mL}^{-1}$ for propranolol as the reference substance [22,23] and 100 and $250 \mathrm{mg} \cdot \mathrm{mL}^{-1}$ for SEV-SBEßCD with transport buffer as the pEND medium or heat-inactivated human serum for an experimental time of $120 \mathrm{~min}$. The amounts of transport substance over time of propranolol and SEV-SBE $\beta C D$ complex under sink conditions $(10 \%$ and $5 \%$ of human serum were presented on apical and basolateral sides, respectively) are displayed in Figure 6. The most rapid increase of the detectible substance on the acceptor side of the system was observed for highly lipophilic $(\log \mathrm{P}=3.56)$ 
propranolol [24] with a passive transcellular route [25] of permeation and cumulative linear distribution (Figure 6A). On the other hand, the SEV-SBE $\beta C D$ complex showed a gradual increase followed by an early onset of a steady state after about $60 \mathrm{~min}$ and then a subsequent decrease of the SEV-SBE $3 C D$ concentration after 80 min according to the non-linear distribution pattern (Figure 6B).
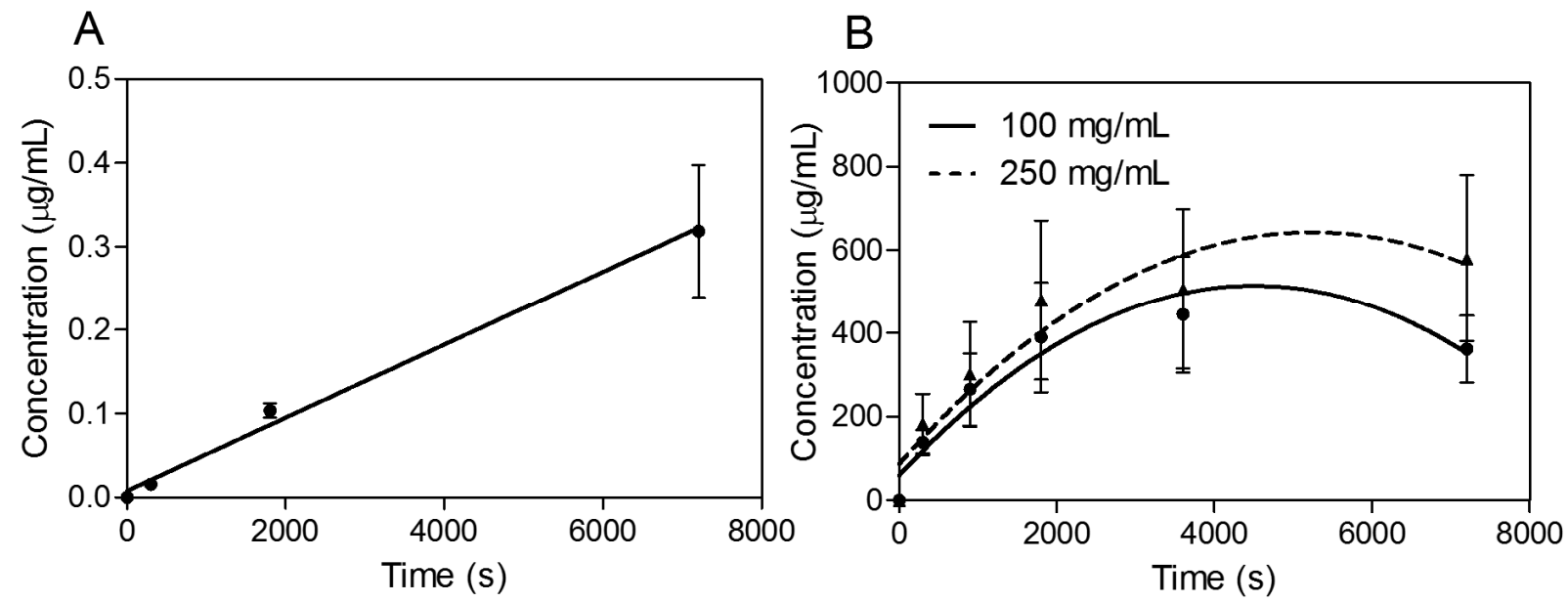

Figure 6. Transwell ${ }^{\circledR}$ BBB transport studies of propranolol as a system validation substance (A) and SEV-SBEßCD (B). Collagen 4-coated empty inserts were used as a control. Second-order polynomial (quadratic) curves are implemented to connect and fit the data points. Data represent means \pm standard deviation of three independent experiments.

Based on these findings, apparent permeability values $\left(\mathrm{P}_{\text {app }}\right)$ were first calculated using the classical approach described in the following equation:

$$
P_{a p p}=\frac{d Q}{d t} \times \frac{V_{b}}{A C_{0}}
$$

where $\frac{d Q}{d t}$ is the slope of the cumulative concentration in the basolateral chamber, $C_{0}$ is the initial concentration of the substance in the apical chamber, $A$ is the diffusion area $\left(A=0.336 \mathrm{~cm}^{2}\right)$, and $V_{b}$ is the volume of the basolateral chamber $\left(V_{b}=0.9 \mathrm{~mL}\right)$. In this formula, the permeability coefficient is dependent on the slope of the function of cumulative quantity absorbed vs. time [26]. As can be seen for SEV-SBE $\beta C D$, its distribution curve was flattened with increasing incubation time. Therefore, only the initial slope was used to calculate $P_{a p p}$ values. According to Hidalgo and co-authors [27], substances with $P_{\text {app }}$ values $>1.0 \times 10^{-6} \mathrm{~cm} \cdot \mathrm{s}^{-1}$ possess high absorption (for Caco-2 cells)/permeation potential, and those with $P_{a p p}$ values $>2.0 \times 10^{-6} \mathrm{~cm} \cdot \mathrm{s}^{-1}$ indicate a bioavailability of more than $90 \%$ [28]. The $P_{a p p}$ values for SEV-SBE $\beta C D$ were calculated in the range of $12.32 \times 10^{-6}\left(100 \mathrm{mg} \cdot \mathrm{mL}^{-1}\right)$ to $6.54 \times 10^{-6} \mathrm{~cm} \cdot \mathrm{s}^{-1}$ $\left(250 \mathrm{mg} \cdot \mathrm{mL}^{-1}\right)$ possessing much higher $\mathrm{BBB}$ permeation rates than the reference substance $\left(P_{a p p}=3.93 \times 10^{-6} \mathrm{~cm} \cdot \mathrm{s}^{-1}\right)$. Considering this high permeability together with the high lipophilicity of $\mathrm{SEV}$, a passive transcellular uptake route might be postulated. It can also be hypothesized from the in vitro BBB permeability experiments that sevoflurane formulation most likely changes the disposition of the anesthetic in the body after intravenous injection, providing its accumulation in the brain, presumably as a result of the CD competing for drug binding with human plasma proteins [29]. Apart from that, there was a significant difference in the apparent permeability of the complexed drug at low 
and high concentrations. This was likely a result of the SEV's volatile nature (which is the cause of the high deviation rate) along with the relatively high $\delta$ blood/gas value of 0.69 [2] and a limited number of pores in the PET membrane serving as a restricting factor.

Due to the random process of the hydroxyl group substitution in the SBE $\beta C D$ molecule with the sulfobutyl ether groups, the manufacturer has not determined the actual substitution pattern and molecular spatial configurations. Therefore, the $\mathrm{SBE}_{7} \beta \mathrm{CD}$ isomer with all of the sulfobutyl ether groups grafted to 6-OH (primary hydroxyl groups) on the glucose subunit was considered to ensure sufficient steric hindrance, as implemented in the previous molecular studies [7,30,31]. As reported by Shityakov and co-authors $[32,33]$, substances with $\Delta \mathrm{G}_{\text {bind }}$ values $\leq-6.0 \mathrm{kcal} \cdot \mathrm{mol}^{-1}$ possess high binding affinities to the host molecule, and those with $\Delta \mathrm{G}_{\text {bind }}$ values $\geq-6.0 \mathrm{kcal} \cdot \mathrm{mol}^{-1}$ indicate low binding modes. Therefore, using the AutoDock program, the calculations for SEV-SBE7 $\beta C D$ with a 1:1 stoichiometry provided an extremely low binding affinity to very hydrophilic $\mathrm{SBE}_{7} \beta \mathrm{CD}$ ( $\left.\log \mathrm{P}_{\mathrm{MLP}}=-18.03\right)$, detected for sevoflurane with an average $\Delta \mathrm{G}_{\text {bind }}$ value of $-1.727 \pm 0.042 \mathrm{kcal} \cdot \mathrm{mol}^{-1}$ (3 top poses) and an average binding constant $\left(\mathrm{K}_{\mathrm{b}}\right)$ of $53.66 \pm 9.24 \mathrm{mM}$, enhancing drug liberation from the cyclodextrin amphiphilic cavity. A tendency toward rapid SEV liberation was observed in the experiment, where sevoflurane in the SEV-SBE $\beta C D$ complex was completely released from the aqueous solution within 60 min. The lipid dispersion using SMOFlipid microemulsion (Fresenius Kabi), meanwhile, trapped more than 50\% of the drug even after $2 \mathrm{~h}$ of incubation at body temperature (unpublished data).

An MLP-based parameter, called the lipophilicity index $(L I)$, was used to evaluate the lipophilicity of cyclodextrin pockets, according to the equation shown below [34]:

$$
L I=\frac{\left|\sum M L P^{+}\right|}{\left|\sum M L P^{+}\right|+\left|\sum M L P^{-}\right|} \times 100 \%
$$

where $\sum M L P^{+}$and $\sum M L P^{-}$are the sum of the MLP values assigned to each hydrophobic fitting points bearing either a hydrophobic or polar potential. A color-coded MLP visualization of SBE7 $\beta C D$ $\left(\log \mathrm{P}_{\mathrm{MLP}}=-18.03\right)$ as shown in Figure 7 was generated using MLPTools, with the results indicating a mild hydrophilic/hydrophobic differentiation between the "inner" and "outer" sides of the molecule. Due to the location of the secondary hydroxyl groups in the second and third positions of each D-glucopyranosyl residue, sulfo groups with the other ether linkers (-O-) and the oxygen atoms involved in the $\alpha-1,4$ glycosidic bond linkages, this CD surface region becomes highly hydrophilic. Contrary to this, the buried areas of the $\mathrm{CD}$ molecule (i.e., the blue and yellow regions) are mainly associated with the D-glucopyranosyl residues and only partially with butyl fragments, contributing to a more clearly amphiphilic/lipophilic surface (Figure 7A). It is evident from part B of Figure 7 that highly lipophilic SEV is deeply embedded in the $\mathrm{SBE}_{7} \beta \mathrm{CD}$ cavity with its fluoromethoxy and trifluoromethyl groups oriented towards the primary face.

Finally, the $\log \mathrm{P}_{\mathrm{MPL}}-$ associated search of $100 \mathrm{SEV}$ spatial conformations $\left(\log \mathrm{P}_{\mathrm{MLP}}=2.85 \pm 0.04\right)$ indicated no significant change in the distribution of lipophilicity following the drug-CD complexation (Figure 7C). By considering the $L I$ threshold for polar $(L I<10 \%)$ and non-polar $(L I>10 \%)$ binding sites [34], the SEV-SBE7 $\beta C D$ complex contained amphiphilic center-oriented pocket mainly characterized by its polar contribution to the complexation with the $L I$ value of $2.79 \%$ diminishing the overall host-guest affinity (Figure 7D). 


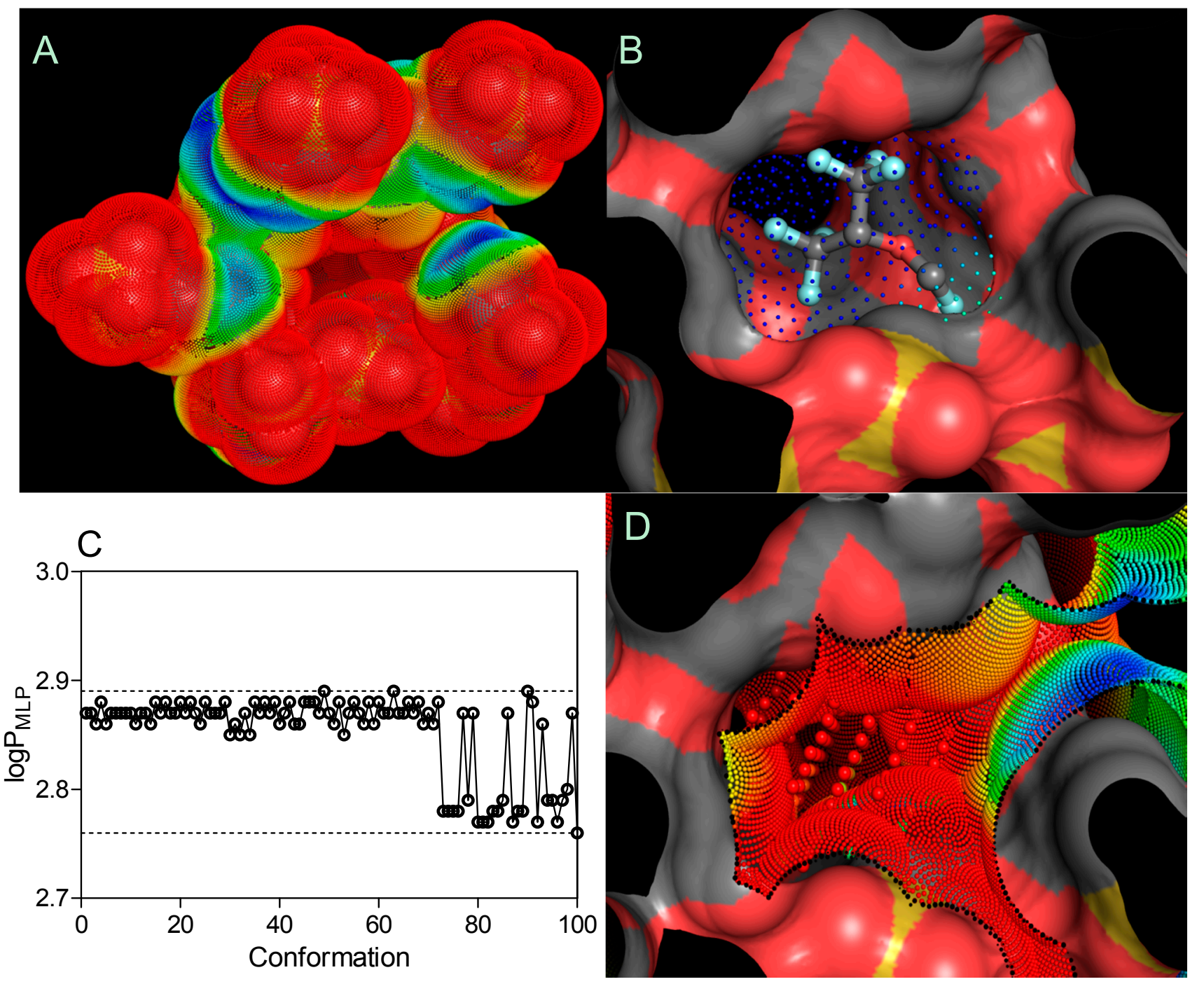

Figure 7. Three-dimensional models of the $\mathrm{SBE}_{7} \beta C D(\mathbf{A})$ structure and $\mathrm{SEV}-\mathrm{SBE}_{7} \beta \mathrm{CD}$ (B) complex (obtained by molecular docking) with MLP-surfaces around the cyclodextrin molecule and best conformational pose (cluster with RMSD values of $2 \AA$ ) of the ligand. $\log P_{M L P}(\mathbf{C})$ and MLP-Pocket (D) display the distribution of lipophilicity for the SBE $7 \beta C D$ binding site and pose conformations of sevoflurane. The thresholds are depicted as dashed lines. MLP calculated as hydrophobic fitting points depicted in smaller colored spheres according to the MLP range from red (the most polar points) to blue (the most hydrophobic points). MLP-pocket in the center of the center of $\mathrm{SBE}_{7} \beta \mathrm{CD}$ molecule is depicted in bigger colored spheres. Molecular surface was calculated to visualize the cyclodextrin cavity. The ball-and-stick SEV model is colored according to its atomic composition. Hydrogen atoms are omitted to enhance clarity.

\section{Experimental Section}

\subsection{Chemicals and Optimized Formulation Procedure}

Sevoflurane (ID: 70435VA) was obtained from Abbott Laboratories (Abbott GmbH, Wiesbaden, Germany), and SBEßCD (ID: CYL-3666) was produced by CycloLab (Budapest, Hungary) as the 
starting materials for complexation. The SEV-SBE $\beta C D$ complex with $8.6 \%$ [w\%] sevoflurane relative content was prepared according to the optimized formulation procedure: SBE $\beta C D(22.8 \mathrm{~g})$ was dissolved in freshly distilled water $(190 \mathrm{~mL})$ in a round-bottom flask, yielding a clear solution. The SBE $\beta C D$ substance was cooled down to $8{ }^{\circ} \mathrm{C}$. Then, sevoflurane $(5.1 \mathrm{~g})$ was added, the flask was closed tightly (stoppered), and its contents were stirred at 400 RPM for $3 \mathrm{~h}$. Finally, the SEV-SBEßCD solubilized solution was obtained in a single homogenous liquid phase and the total amount of solution was frozen and lyophilized.

\subsection{Gas Chromatography and X-ray Diffraction Studies}

Head-space Shimadzu GC-17A gas chromatography (Shimadzu Europa GmbH, Duisburg, Germany) was performed by using a flame ionization detector for the quantitative determination of drug substance in the SEV-SBE $\beta C D$ complex. After incubation for $10 \mathrm{~min}$ at $60^{\circ} \mathrm{C}$, a $250-\mu \mathrm{L}$ sample of the vapor was injected into the gas chromatograph with a syringe at $70{ }^{\circ} \mathrm{C}$. The $\mathrm{X}$-powder diffraction investigations were performed by using standard normal $\mathrm{CuK}_{\alpha}$ radiation. The reflection peaks were registered in the $2 \theta$ angle range at $5-40$ degrees.

\subsection{Polarized Light Microscopy}

Microscopic observation of the SEV-SBE $\beta C D$ structure was carried out under an Ergaval Zeiss Jena binocular microscope (VEB Carl Zeiss JENA, Jena, Germany) equipped with a $32 \mathrm{~mm}$ polarizing filter.

\subsection{Capillary Electrophoresis}

Capillary electrophoresis was performed with an Agilent Capillary Electrophoresis 3DCE system (Agilent Technologies, Santa Clara, CA, USA) on a $50 \mathrm{~cm}, 50 \mu \mathrm{m}$ uncoated fused-silica capillary. The buffer contained $30 \mathrm{mM}$ benzoic acid and $100 \mathrm{mM}$ TRIS at a $\mathrm{pH}$ between 8.3 and 8.7. Linear ramp voltage from 0 to $30 \mathrm{kV}$ was applied for $0-10$ min intervals; then, $30 \mathrm{kV}$ was set for $10-30 \mathrm{~min}$ interval. An indirect detection mode was applied using a $350 \mathrm{~nm}$ signal and a $200 \mathrm{~nm}$ reference detector with $20 \mathrm{~nm}$ of bandwidth.

\subsection{Cell Toxicity and in Vitro Transport Studies}

Mouse primary brain microvascular endothelial (pEND) cells (Pelobiotech GmbH, Martinsried, Germany) were seeded on collagen IV-coated Transwell ${ }^{\circledR}$ filters with $0.4 \mu \mathrm{m}$ pores (Greiner Bio-One $\mathrm{GmbH}$, Frickenhausen, Germany) or 96-welled plates and cultured using mouse endothelial cell medium (Cell Biologics, Chicago, IL, USA) with a penicillin/streptomycin mixture. The cells were grown to confluence for 2 weeks. Next, the medium in the apical chamber was replaced with a fresh medium containing $10 \%$ human serum and SEV-SBE $\beta C D$ in a concentration of 100 and $250 \mathrm{mg} \cdot \mathrm{mL}^{-1}$. The high SEV-SBE $\beta C D$ concentrations were used due to low LOD (limit of detection) parameter in the $\mu \mathrm{g} \cdot \mathrm{mL}^{-1}$ range for sevoflurane and its extreme volatility. Meanwhile, the medium in the basolateral chamber was replaced with a fresh medium containing 5\% human serum. $100 \mu \mathrm{L}$ samples were taken from the basolateral chamber after 5, 15, and $30 \mathrm{~min}$, and 1 and $2 \mathrm{~h}$, each time with fresh medium 
replacement. For the toxicity assay, the cells were incubated with SEV-SBE $\beta C D$ in a concentration from $46.5 \mu \mathrm{g} \cdot \mathrm{mL}^{-1}$ to $46.5 \mathrm{mg} \cdot \mathrm{mL}^{-1}$ and propranolol hydrochloride (Sigma-Aldrich Chemie $\mathrm{GmbH}$, Munich, Germany) as a reference substance in a concentration of $30 \mu \mathrm{g} \cdot \mathrm{mL}^{-1}$ for $24 \mathrm{~h}$ at $37{ }^{\circ} \mathrm{C}$. Afterward, cell viability was assessed using the CellTiter-Glo ${ }^{\circledR}$ luminescent cell viability assay (Promega, Madison, WI, USA) kit according to the manufacturer's instructions. Briefly, the test compound and controls were added to the cells, and after the intended incubation period, 30-min incubation at room temperature followed. The CellTiter-Glo ${ }^{\circledR}$ solution was then added. Lysis was induced for 2 min with shaking, followed by a 10-min equilibration at room temperature. Luminescence and fluorescence (4 kDa FITC-labeled dextran permeation assay) were read using the Tecan GENios Microplate Reader (MTX Lab Systems, Inc., Vienna, VA, USA). Transendothelial electric resistance (TER) of the cell monolayer was measured using a TER voltohmmeter (World Precision Instruments, Sarasota, FL, USA) before and after the experiment. The TER values of blank filters, coated with collagen IV, were used as a control.

\subsection{Clean-Up and Liquid Chromatography Coupled by Mass Spectroscopy}

The clean-up approach with serum was mixing the sample with two volumes of ice cold methanol to precipitate the proteins, followed by centrifugation at $4{ }^{\circ} \mathrm{C}$ with $10,000 \mathrm{RPM}$ for $10 \mathrm{~min}$ and analysis of the supernatant. For the quantitative determination of SEV in the samples of the in vitro $\mathrm{BBB}$ transport, a combined method was developed using high performance liquid chromatography with tandem mass spectrometric detection. The analyte was separated on a Kinetex PFP, $100 \AA, 100 \times 2.1$ mm, i.d.; $1.7 \mu \mathrm{m}$ particle size UHPLC column (Phenomenex, Aschaffenburg, Germany) with a mobile phase consisting of methanol and water at a flow rate of $0.3 \mathrm{~mL} \cdot \mathrm{min}^{-1}$, and the column oven was set to $40{ }^{\circ} \mathrm{C}$. Injection volume was $5 \mu \mathrm{L}$ of the supernatant. A gradient elution was performed with eluent $\mathrm{A}$ (water) and eluent B (methanol): 0 min, 55\% B; $0.5 \mathrm{~min}, 55 \% \mathrm{~B} ; 2.5 \mathrm{~min}, 95 \% \mathrm{~B} ; 3.5 \mathrm{~min}, 99 \% \mathrm{~B}$; $3.51 \mathrm{~min}, 55 \% \mathrm{~B} ; 4.5 \mathrm{~min}$, stop run. The ionization reagent $(0.05 \%$ ammonia $)$ was delivered post column by means of a second HPLC pump and a T-piece. Detection was achieved by a Shimadzu 8030-Plus mass spectrometer (Shimadzu Europa GmbH) abbreviated as LC-MS/MS set at unit resolution in the multiple reaction monitoring mode. Atmospheric pressure chemical ionization (APCI) was used for ion production. The mean recovery for SEV was $95 \%$, with a lower limit of quantification set at $2.5 \mu \mathrm{g} \cdot \mathrm{mL}^{-1}$. The LabSolution 5.60 SP2 software (Shimadzu Corporation, Kyoto, Japan) was used to control the LC-MS/MS system and to perform analyses.

\subsection{Molecular Modeling Studies}

The 3D coordinates of sevoflurane structure were retrieved from the PubChem database (Figure 8). Since no 3D structure for sulfobutyl-ether7- $\beta$-cyclodextrin $\left(\mathrm{SBE}_{7} \beta \mathrm{CD}\right)$ was available, the molecule was constructed and minimized with the Molecular Operating Environment (MOE 2009.10) software (Chemical Computing Group Inc., Montreal, QC, Canada). 


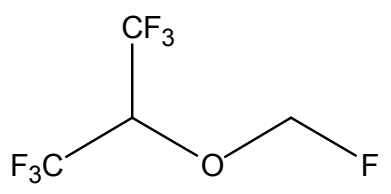

Sevoflurane

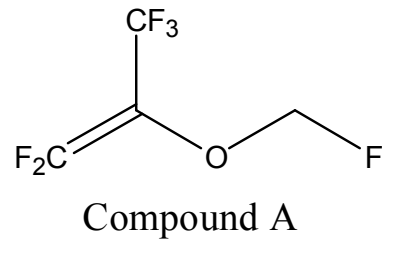

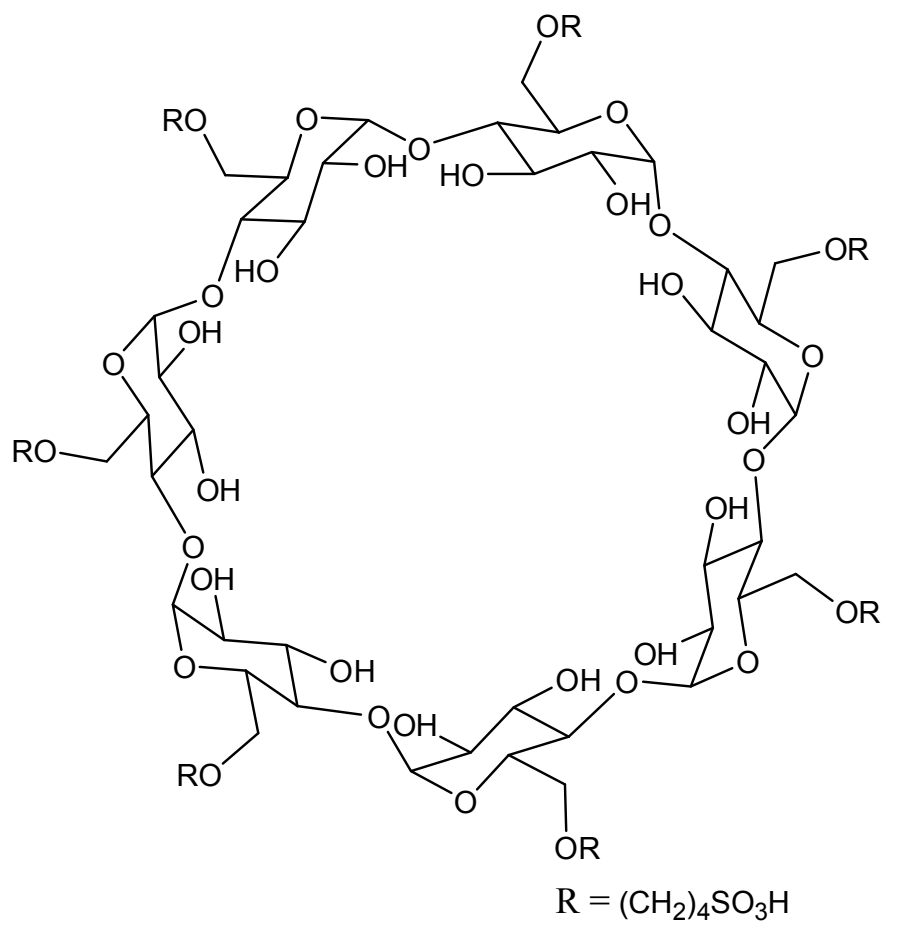

Sulfobutyl-ether ${ }_{7}-\beta$-cyclodextrin

Figure 8. Schematic representations of sevoflurane, compound A, and sulfobutyl-ether7- $\beta$ cyclodextrin $\left(\mathrm{SBE}_{7} \beta \mathrm{CD}\right)$ molecules.

The host and guest structure preparations for molecular docking included Gasteiger partial charges assignment [35] and rotatable bonds definition. Rigid-flexible molecular docking was applied to the center of cyclodextrin using Cartesian coordinates: $\mathrm{x}=56.49 \AA, \mathrm{y}=12.97 \AA$, and $\mathrm{z}=9.29 \AA$. AutoDock v.4.2.5.1 [36] integrated in the PyMol AutoDock/Vina plugin [37] was used in the study. The grid spacing of $0.375 \AA$ with a dimension size of $30 \AA$ was used to create the grid maps. In order to increase a conformational sampling of the drug, a number of standard genetic algorithm dockings (ga_run) was set to 100 [38]. Docking output results were represented by the approximation function as the estimated Gibbs free energy of binding ( $\Delta \mathrm{G}_{\text {bind }}$ ). The molecular lipophilicity potential (MLP), based on experimental octanol/water partition ( $\log \mathrm{P})$ coefficients [39], was determined by the MLP tools-A PyMol plugin [40] using the following general equation [34]:

$$
M L P=\sum_{i=1}^{N} f_{i} f c t\left(d_{i k}\right)
$$

where $i$ is the label of the molecular fragment, $N$ is the total number of fragments in the molecule, $f_{i}$ is the lipophilic constant of fragment, $f_{c} t$ is the distance function, and $d_{i k}$ is the distance between fragment $i$ and space point $k$. The overall sum of polar and hydrophobic points of a MLP surface allows a back calculation of its experimental parameter origin, the $\log \mathrm{PMLP}_{\mathrm{ML}}[40]$ :

$$
\log P_{M L P}=\sum M L P^{+} w^{+}+\sum M L P^{-} w^{-}+C
$$

where $M L P^{-}$and $M L P^{+}$are the polar and hydrophobic parts of the MLP, respectively. The weighting factor $w^{+}$and $w^{-}$as well as correlation coefficient $C$ have been optimized on a set of molecular structures with experimentally determined $\log \mathrm{P}$ values. 


\subsection{Graphic Representation and 3D Animation}

All molecular rendering scenes, graphic representations, and 3D animation were prepared with the PyMol molecular graphics system (Schrödinger, LLC, San Diego, CA, USA), Wolfram Mathematica 10 (The Wolfram Centre, Long Hanborough, UK), and GraphPad prism v.4 for Windows software (GraphPad Software, Inc., San Diego, CA, USA). All the data are represented as the means \pm standard deviations.

\section{Conclusions}

Our results indicate that it is possible to prepare a SEV-SBE $\beta C D$ inclusion complex with high chemical stability using an industrially feasible optimized formulation method. Cell viability tests did not detect any signs of toxicity of the complex on primary cerebral endothelial cells (pEND). The inclusion complex exhibited a significantly higher BBB permeation profile as compared with the reference substance (propranolol) concerning calculated apparent permeability values $\left(P_{\text {app }}\right)$ in the range of $12.32 \times 10^{-6}$ to $6.54 \times 10^{-6} \mathrm{~cm} \cdot \mathrm{s}^{-1}$ at 100 and $250 \mathrm{mg} \cdot \mathrm{mL}^{-1}$ concentration possessing much higher BBB permeation rates than that of the reference substance $\left(P_{a p p}=3.93 \times 10^{-6} \mathrm{~cm} \cdot \mathrm{s}^{-1}\right)$. Taking into account this high permeability together with the high lipophilicity of SEV, a passive transcellular uptake route can be speculated. Finally, SEV binding affinity to SBE $\beta C D$ was confirmed by a minimal Gibbs free energy of binding ( $\Delta \mathrm{G}_{\text {bind }}$ ) value of $-1.727 \pm 0.042 \mathrm{kcal} \cdot \mathrm{mol}^{-1}$ and an average binding constant $\left(\mathrm{K}_{\mathrm{b}}\right)$ of $53.66 \pm 9.24 \mathrm{mM}$, enhancing drug liberation from the cyclodextrin amphiphilic cavity. Overall, the SEV-SBE$\beta C D$ complex has the potential to be used in clinical applications as an injectable formulation for controlled drug delivery.

\section{Supplementary Materials}

Supplementary materials can be accessed at: http:/www.mdpi.com/1420-3049/20/06/10264/s1.

\section{Acknowledgments}

Special thanks are extended to Anna Poon from the City College of New York for her assistance in paper writing. The authors are grateful to the IZKF (Interdisziplinäres Zentrum für Klinische Forschung der Universität Würzburg) and the BMBF (Bundesministerium für Bildung und Forschung) for the support of this work by providing grants (BMBF01, EO1004) to Carola Förster.

\section{Author Contributions}

S.S., J.-A.B., N.R. and C.F. participated in the research design. S.S., I.P., K.P. and E.S. performed the experiments and analyzed the data. The manuscript was written by S.S. All authors read and approved the final manuscript.

\section{Conflicts of Interest}

The authors declare no conflict of interest. 


\section{References}

1. Wallin, R.F.; Regan, B.M.; Napoli, M.D.; Stern, I.J. Sevoflurane: A new inhalational anesthetic agent. Anesth. Analg. 1975, 54, 758-766.

2. Ebert, T.J.; Schmid, P.G., III. Inhalation Anesthesia, Chapter 15. In Clinical Anesthesia, 4th ed.; Barash, P.G., Cullen, B.F., Stoelting, R.K., Eds.; Lippincott Williams\& Wilkins: Philadelphia, PA, USA, 2001; pp. 377-387.

3. Jin, L.; Baillie, T.A.; Davis, M.R.; Kharasch, E.D. Nephrotoxicity of sevoflurane compound A [fluoromethyl-2,2-difluoro-1-(trifluoromethyl)vinyl ether] in rats: Evidence for glutathione and cysteine conjugate formation and the role of renal cysteine conjugate beta-lyase. Biochem. Biophys. Res. Commun. 1995, 210, 498-506.

4. Martin, J.L.; Kandel, L.; Laster, M.J.; Kerschmann, R.L.; Eger, E.I., II. Studies of the mechanism of nephrotoxicity of compound A in rats. J. Anaesth. 1997, 11, 32-37.

5. Ma, D.Q.; Rajewski, R.A.; Stella, V.J. New injectable melphan formulations utilizing (SBE)7m-b-CD or HP-b-CD. Int. J. Pharm. 1999, 189, 227-234.

6. Loftsson, T.; Brewster, M.E. Pharmaceutical applications of cyclodextrins. 1. Drug solubilization and stabilization. J. Pharm. Sci. 1996, 85, 1017-1025.

7. Jain, A.S.; Date, A.A.; Pissurlenkar, R.R.; Coutinho, E.C.; Nagarsenker, M.S. Sulfobutyl ether(7) beta-cyclodextrin (SBE(7) beta-CD) carbamazepine complex: Preparation, characterization, molecular modeling, and evaluation of in vivo anti-epileptic activity. AAPS PharmSciTech 2011, $12,1163-1175$.

8. Nagase, Y.; Hirata, M.; Arima, H.; Tajiri, S.; Nishimoto, Y.; Hirayama, F.; Irie, T.; Uekama, K. Protective effect of sulfobutyl ether beta-cyclodextrin on DY-9760e-induced hemolysis in vitro. J. Pharm. Sci. 2002, 91, 2382-2389.

9. Tötterman, A.M.; Schipper, N.G.; Thompson, D.O.; Mannermaa, J.P. Intestinal safety of water-soluble beta-cyclodextrins in paediatric oral solutions of spironolactone: Effects on human intestinal epithelial Caco-2 cells. J. Pharm. Pharmacol. 1997, 49, 43-48.

10. Irie, T.; Uekama, K. Pharmaceutical applications of cyclodextrins. III. Toxicological issues and safety evaluation. J. Pharm. Sci. 1997, 86, 147-162.

11. Kiss, T.; Fenyvesi, F.; Bácskay, I.; Váradi, J.; Fenyvesi, E.; Iványi, R.; Szente, L.; Tósaki, A.; Vecsernyés, M. Evaluation of the cytotoxicity of beta-cyclodextrin derivatives: Evidence for the role of cholesterol extraction. Eur. J. Pharm. Sci. 2010, 40, 376-380.

12. McIntosh, M.P.; Schwarting, N.; Rajewski, R.A. In vitro and in vivo evaluation of a sulfobutyl ether beta-cyclodextrin enabled etomidate formulation. J. Pharm. Sci. 2004, 93, 2585-2594.

13. Babu, M.K.; Godiwala, T.N. Toward the development of an injectable dosage form of propofol: Preparation and evaluation of propofol-sulfobutyl ether 7-beta-cyclodextrin complex. Pharm. Dev. Technol. 2004, 9, 265-275.

14. McIntosh, M.P.; Narita, H.; Kameyama, Y.; Rajewski, R.A.; Goto, H. Evaluation of mean arterial blood pressure, heart rate, and sympathetic nerve activity in rabbits after administration of two formulations of etomidate. Vet. Anaesth. Analg. 2007, 34, 149-156. 
15. Goodchild, C.S.; Serrao, J.M.; Kolosov, A.; Boyd, B.J. Alphaxalone Reformulated: A Water-Soluble Intravenous Anesthetic Preparation in Sulfobutyl-Ether-beta-Cyclodextrin. Anesth. Analg. 2014, doi:10.1213/ANE.0000000000000559.

16. Ribeiro, A.; Figueiras, A.; Santos, D.; Veiga, F. Preparation and solid-state characterization of inclusion complexes formed between miconazole and methyl-beta-cyclodextrin. AAPS PharmSciTech 2008, 9, 1102-1109.

17. Naidu, N.B.; Chowdary, K.P.; Murthy, K.V.; Satyanarayana, V.; Hayman, A.R.; Becket, G. Physicochemical characterization and dissolution properties of meloxicam-cyclodextrin binary systems. J. Pharm. Biomed. Anal. 2004, 35, 75-86.

18. Pomponio, R.; Gotti, R.; Fiori, J.; Cavrini, V.; Mura, P.; Cirri, M.; Maestrelli, F. Photostability studies on nicardipine-cyclodextrin complexes by capillary electrophoresis. J. Pharm. Biomed. Anal. 2004, 35, 267-275.

19. Hong, J.; Shah, J.C.; Mcgonagle, M.D. Effect of cyclodextrin derivation and amorphous state of complex on accelerated degradation of ziprasidone. J. Pharm. Sci. 2011, 100, 2703-2716.

20. Fischer, K. Neues Verfahren zur maßanalytischen Bestimmung des Wassergehaltes von Flüssigkeiten und festen Körpern. Angew. Chem. 1935, 48, 394-396.

21. Nitzschke, R.; Wilgusch, J.; Kersten, J.F.; Trepte, C.J.; Haas, S.A.; Reuter, D.A.; Goetz, A.E.; Goepfert, M.S. Changes in sevoflurane plasma concentration with delivery through the oxygenator during on-pump cardiac surgery. Br. J. Anaesth. 2013, 110, 957-965.

22. Nagare, N.; Damre, A.; Singh, K.S.; Mallurwar, S.R.; Iyer, S.; Naik, A.; Chintamaneni, M. Determination of site of absorption of propranolol in rat gut using in situ single-pass intestinal perfusion. Indian J. Pharm. Sci. 2010, 72, 625-629.

23. Imam, S.S.; Ahad, A.; Agil, M.; Sultana, Y.; Ali, A. A validated RP-HPLC method for simultaneous determination of propranolol and valsartan in bulk drug and gel formulation. J. Pharm. Bioallied Sci. 2013, 5, 61-65.

24. Dutta, H.; Sengupta, M.; Pal, D.K.; De, A.U.; Sengupta, C. Effect of propranolol hydrochloride on blood cell lipids in relation to partition coefficient and biological activity. Indian J. Biochem. Biophys. 1993, 30, 128-132.

25. Walgren, R.A.; Walle, T. The influence of plasma binding on absorption/exsorption in the Caco-2 model of human intestinal absorption. J. Pharm. Pharmacol. 1999, 51, 1037-1040.

26. Heinlein, A.; Metzger, M.; Walles, H.; Buettner, A. Transport of hop aroma compounds across Caco-2 monolayers. Food Funct. 2014, 5, 2719-2730.

27. Hidalgo, I.; Li, J. The evolving role of the Caco-2 cell model to estimate intestinal absorption potential and elucidate transport mechanisms. In The Process of New Drug Discovery and Development, 2nd ed.; Smith, C.G., O’Donnell, J.T., Eds.; Informa Healthcare USA, Inc.: New York, NY, USA, 2006; pp. 161-186.

28. Biganzoli, E.; Cavenaghi, L.A.; Rossi, R.; Brunati, M.C.; Nolli, M.L. Use of a Caco-2 cell culture model for the characterization of intestinal absorption of antibiotics. Il Farmaco 1999, 54, 594-599.

29. Frijlink, H.W.; Franssen, E.J.; Eissens, A.C.; Oosting, R.; Lerk, C.F.; Meijer, D.K. The effects of cyclodextrins on the disposition of intravenously injected drugs in the rat. Pharm. Res. 1991, 8 , 380-384. 
30. Blach, P.; Landy, D.; Fourmentin, S.; Surpateanu, G.; Bricout, H.; Ponchel, A.; Hapiot, F.; Monflier, E. Sulfobutyl ether-beta-cyclodextrins: Promising supramolecular carriers for aqueous organometallic catalysis. Adv. Synth. Catal. 2005, 347, 1301-1307.

31. Jug, M.; Mennini, N.; Melani, F.; Maestrelli, F.; Mura, P. Phase solubility, ${ }^{1} \mathrm{H}$ NMR and molecular modelling studies of bupivacaine hydrochloride complexation with different cyclodextrin derivates. Chem. Phys. Lett. 2010, 500, 347-354.

32. Shityakov, S.; Broscheit, J.; Förster, C. alpha-Cyclodextrin dimer complexes of dopamine and levodopa derivatives to assess drug delivery to the central nervous system: ADME and molecular docking studies. Int. J. Nanomed. 2012, 7, 3211-3219.

33. Shityakov, S.; Forster, C. In silico predictive model to determine vector-mediated transport properties for the blood-brain barrier choline transporter. Adv. Appl. Bioinform. Chem. 2014, 7, 23-36.

34. Nurisso, A.J.; Bravo, J.; Carrupt, P.A.; Daina, A. Molecular docking using the molecular lipophilicity potential as hydrophobic descriptor: Impact on GOLD docking performance. J. Chem. Inf. Model. 2012, 52, 1319-1327.

35. Gasteiger, J.; Marsili, M. Iterative Partial Equalization of Orbital Electronegativity-A Rapid Access to Atomic Charges. Tetrahedron 1980, 36, 3219-3228.

36. Goodsell, D.S.; Morris, G.M.; Olson, A.J. Automated docking of flexible ligands: Applications of AutoDock. J. Mol. Recognit. 1996, 9, 1-5.

37. Seeliger, D.; de Groot, B.L. Ligand docking and binding site analysis with PyMOL and Autodock/Vina. J. Comput. Aided Mol. Des. 2010, 24, 417-422.

38. Shityakov, S.; Sohajda, T.; Puskás, I.; Roewer, N.; Förster, C.; Broscheit, J.A. Ionization states, cellular toxicity and molecular modeling studies of midazolam complexed with trimethyl-betacyclodextrin. Molecules 2014, 19, 16861-16876.

39. Gaillard, P.; Carrupt, P.A.; Testa, B.; Boudon, A. Molecular lipophilicity potential, a tool in 3D QSAR: Method and applications. J. Comput. Aided Mol. Des. 1994, 8, 83-96.

40. Oberhauser, N.; Nurisso, A.; Carrupt, P.A. MLP Tools: A PyMOL plugin for using the molecular lipophilicity potential in computer-aided drug design. J. Comput. Aided. Mol. Des. 2014, 28, 587-596.

Sample Availability: Samples of the compounds are not available.

(C) 2015 by the authors; licensee MDPI, Basel, Switzerland. This article is an open access article distributed under the terms and conditions of the Creative Commons Attribution license (http://creativecommons.org/licenses/by/4.0/). 